\title{
Delegate MAS for Large Scale and Dynamic PDP: A Case Study
}

\author{
Shaza Hanif, Rinde R.S. van Lon, Ning Gui, and Tom Holvoet
}

\begin{abstract}
Pickup and Delivery Problems (PDPs) have received significant research interest in the past decades. Their industrial relevance has stimulated the study of various types of solutions. Both centralized solutions, using discrete optimization techniques, as well as distributed, multi-agent system (MAS) solutions, have proven their merits. However, real PDP problems today are more and more characterized by (1) dynamism - in terms of tasks, service time, vehicle availability, infrastructure availability, and (2) their large scale - in terms of the geographical field of operation, the number of pickup and delivery tasks and vehicles. A combination of both characteristics brings unsolved challenges.

Delegate MAS is a coordination mechanism that could prove to be valuable for constructing a decentralized solution for dynamic and large scale PDP problems. In this paper, we illustrate a solution based on delegate MAS for solving PDP. Our solution enables different agents to dynamically collect and disseminate local information and make decisions in a fully decentralized way. We applied our approach to a concrete case study. Experimental results indicate the suitability of the approach for dynamic and large scale PDP problems.
\end{abstract}

\section{Introduction}

Pickup and Delivery Problems (PDPs) [4] constitute a well-known family of optimization problems in logistics, faced by package transportation companies. In a PDP, loads have to be transported form origins to destinations by a set of vehicles. Many approaches have been proposed for addressing numerous variants of the problem for more than half a century [4, 5].

Shaza Hanif · Rinde R.S. van Lon · Ning Gui · Tom Holvoet
Katholiek Universiteit Leuven , Dept. Computer Science,
Celestijnenlaan 200A, B-3001 Heverlee
e-mail: \{Shaza.Hanif, Rinde.vanLon, Ning.Gui, Tom.Holvoet\} @
CS.kuleuven be

F.M.T. Brazier et al. (Eds.): Intelligent Distributed Computing V, SCI 382, pp. 2333

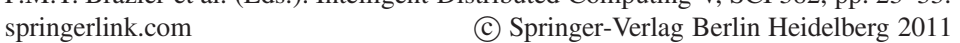


Traditional approaches tackle the problem by applying discrete optimization approaches (DOA) [20]. These approaches require formulating the problem in a mathematical model, in which constraints and objective functions are mathematically expressed. These yield static solutions; when the problem changes, the mathematical model needs updating, and potentially the selection of the DOA to solve the problem needs to be reconsidered.

In practice, a PDP is typically both a dynamic and large scale problem. New transportation requests might arrive, accidents or traffic jams may require vehicles to change their schedules, service time may vary. Such dynamism cannot be handled effectively by a solution based on a DOA. Additionally, the scalability requirement creates extra challenges - managing a PDP for large numbers of vehicles, packages in a large geographical environment add considerable stress to any solution.

In order to handle such a dynamic environment in a scalable way, improvements of DOA, often known as on-line algorithms [6, 7], have been proposed. These algorithms use smart heuristics and exploit the problem structure for fast computation times to yield a feasible solution. Powel et al. highlights that as these improvements are based on a static solution, when the problem is highly dynamic and large in scale, such solution approaches become infeasible [18].

We argue that a solution to dynamic problems should embrace the dynamism in the environment and consider dynamism as an integral part of the original problem. This is contrary to the DOA, which integrate the dynamism by modifying a static solution. Decentralized, Multi-agent System (MAS) [22] based solutions incorporate dynamism explicitly in more systematic ways. Jennings indicates that 'Agent-based computing has the potential to significantly improve our ability to model, design and build complex, distributed software systems' [19]. These solutions provide modeling abstractions that are decentralized in nature and are more suitable for presenting scalable solutions for real world systems [21].

Delegate MAS [12] is a coordination mechanism that is well suited for coordination and control applications. It takes dynamism as well as large scale as a primary concern. The mechanism is being studied with good results in various domains of coordination and control applications, including traffic [13, 14] and manufacturing control [12]. In this paper, we present a 'delegate MAS' based solution for solving PDP. In our solution the system entities, vehicles and packages, are modeled as agents. The agents coordinate using delegate MAS to explore the environment around them, collecting locally available knowledge. Moreover, the agents disseminate information to those parts of the system for which their information is relevant.

Two distinguished features make the solution suitable for large scale and dynamic PDP: (i) The vehicles and packages are modeled as individual agents that coordinate using locally available information only. This enables a scalable solution since it is not required to maintain accurate global information for coordination. (ii) The delegate MAS allows the solution to cope with dynamic changes through indirect communication via the environment.

The remainder of the paper is structured as follows: Section 2 discusses the related work from two different aspects, DOA and MAS based approaches. Section 
3 and 4 describe our delegate MAS model for solving PDP and the preparatory experiments respectively. Finally, section 5 concludes the paper.

\section{Related Work}

In the last decade, various taxonomical papers have appeared [4, 5, 6, 16], which classify the diverse approaches proposed for solving multiple variants of PDP. Here, we differentiate between DOA, and MAS based approaches for solving PDP.

\subsection{Discrete Optimization Approaches for Solving PDP}

A wide variety of solutions are based on DOA [4,5]. So-called 'exact DOAs', which guarantee optimal solutions, yield high computational complexity. Therefore, other advanced solutions like heuristics, meta-heuristics and hyper-heuristics are being used [20]. All these approaches are very useful for solving static problems but can not effectively handle the continual change in a dynamic problem.

Online algorithms use a combination of DOA approaches for dealing with dynamism [6]. Several approaches solve the problem by manipulating data available at a certain moment in time using a mathematical model. As new data becomes available, instead of recomputing the mathematical model, they re-optimize the solution using (meta-)heuristics [15, 16, 17]. Another meta-heuristics solution for dynamic PDP is the Ant Colony Optimization (ACO) based approach [20]. For instance, Rizoli et al. [8] presents an online ACO algorithm in which dynamic change is incorporated by evaporating old information and reinstating the new change in the schedule.

While DOAs for dynamic PDP practically handle the dynamism in the environment, they have a clearly identifiable role of a dispatcher. They assume that all information about the problem is known, and calculate optimal routes for serving the set of requests available at a certain amount of time. As a centralized approach, DOAs face scalability challenges in terms of number of vehicles, number of transportation requests or geographical expansion.

\subsection{MultiAgent Systems Based Approaches for Solving PDP}

MASs consist of a set of autonomous software entities that collaborate to attain overall system objectives. As such, MASs provide the modeling abstractions for building decentralized solutions [19], without requiring a mathematical model.

Competitive results were obtained for optimization of logistics in industry [1, 2 , 3 , 11].

For solving dynamic PDP, Fisher et al. a MAS based solution [10] in which tasks are allocated to trucks and company agents using an extension of Contract Net Protocol (CNP) [22]. They compute a basic solution using CNP which is significantly improved by using auction protocols [22]. 
Kouki et al. also present an online solution [9] that performs re-optimization of routes (calculated by a mathematical model) for individual vehicle agents. It uses CNP to intelligently deal with dynamic environments. Both solutions are based on re-optimizing the whole solution for adaptability and may not be feasible for highly dynamic environments. In another MAS based solution [3], a centralized dispatcher allocates incoming requests to regional dispatchers. It adapts to the dynamic environment but its centralized nature limits its scalability.

A common disadvantage of above mentioned solutions is their reliance on centrally maintained global information. This means that the role of dispatcher is still evident, despite that MASs do not require to maintain centralized global information [19]. This centralistic approach faces problems in dealing with large scale PDP.

\section{Delegate MAS for PDP}

The term 'delegate MAS' was coined in [12] after the mechanism was studied in context of manufacturing control. Subsequent research [23, 24] provided more insight in the mechanism. Applications of delegate MAS in different domains show that it is an effective, multipurpose agent coordination mechanism [12] 13, 14].

The purpose of this paper is to evaluate the feasibility of a delegate MAS-based solution for providing a scalable, decentralized solution for dynamic PDPs.

\subsection{Problem Description}

In this case study we consider the 'dynamic pickup and delivery problem with time windows' (DPDPTW) [4]. The DPDPTW can be described as follows. New arriving pick-up-and-delivery tasks need to be allocated to a truck that can serve the request. A task is comprised of a pickup and delivery location and a delivery time window. In our problem dynamism is caused by continually arriving requests. Based on Marrow's [21] concept of scalability, we refer to a solution as scalable if the efficiency of the solution remains proportional to the problem scale.

The problem requires to consider the following concerns. First, all customer requests need to be satisfied by delivering the packages without violating time windows. Second, the total distance traveled by the trucks needs to be minimized.

\subsection{A Delegate MAS Solution}

In this section, we describe a decentralized solution for solving DPDPTW. This solution is designed to be adaptive and scalable.

Our solution consists of two types of primary agents: truck agents and package agents. The agents are situated in the road infrastructure (the environment) [25], which offers localized information sharing for agents. Agents can perceive the environment and act based on the locally (in time and space) available information.

Truck agents can travel over the road infrastructure. A truck agent is responsible for finding nearby packages, for acquiring information from package agents 
about possible paths and for selecting a path. A package arrives dynamically and its status may change over time: when its deadline approaches, it becomes a 'critical package', having a higher priority for pick up. In order to maintain updated information, packages are responsible to periodically inform other nearby package agents about package related information, including package destination and time windows. Packages also manage information about feasible delivery paths and make decisions when multiple truck agents want to pick up a certain package.

In order to achieve coordination between different agents, our approach uses several types of delegate MASs to coordinate agents' interactions. A delegate MAS is a light-weight agent, which performs delegated tasks for their primary agent [24]. This design allows the implementation of auxiliary tasks separated from primary agent logic. To distinguish them from the primary agents, situated light-weight agents in a delegate MAS are called ants. In order to enable such ant based coordination between agents, we assume that there are smart devices installed at pickup and delivery location which have computation and communication capabilities. Three different types of delegate MASs are used in our solution.

1. Feasibility Ants. In order to spread local information to other agents, package agents delegate this functionality to a feasibility delegate MAS. Figure 1 shows how they travel to the neighboring destinations of packages within a certain range and leave information that points back to their sender package. By using feasibility ants, package agents can share their information in a decentralized way and construct local package maps (paths) which will be used for other delegate MAS.

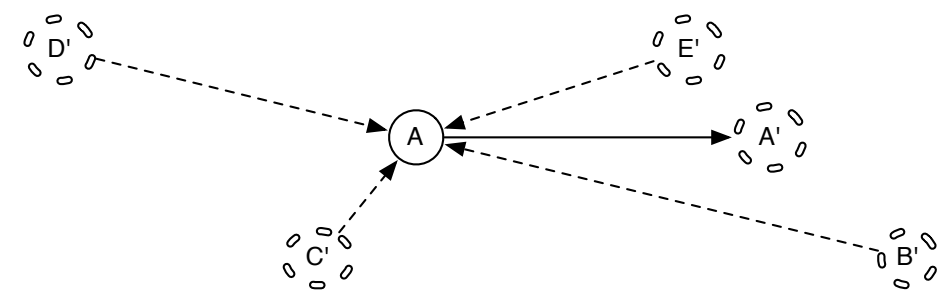

Fig. 1 An abstract representation of behavior of feasibility ants: the package agent A (with destination A') has sent feasibility ants to package destinations B', C', D' and E'. Each of these destinations has information that points back to A.

2. Path Exploration Ants. Path exploration ants are dispatched by truck agents, their goal is to find an optimal delivery path. An exploration ant travels over the network based on the information shared by the feasibility ants. In Figure 2 an example of the behavior of path exploration ants is illustrated.

Typically, path exploration ants are sent out several hops ahead as this reduces the probability of getting stuck in local optima (e.g. going after the package which is closest by). When it reaches a pre-specified number of hops, this ant will return to the truck agent with accumulated information about its path. A truck agent sends 


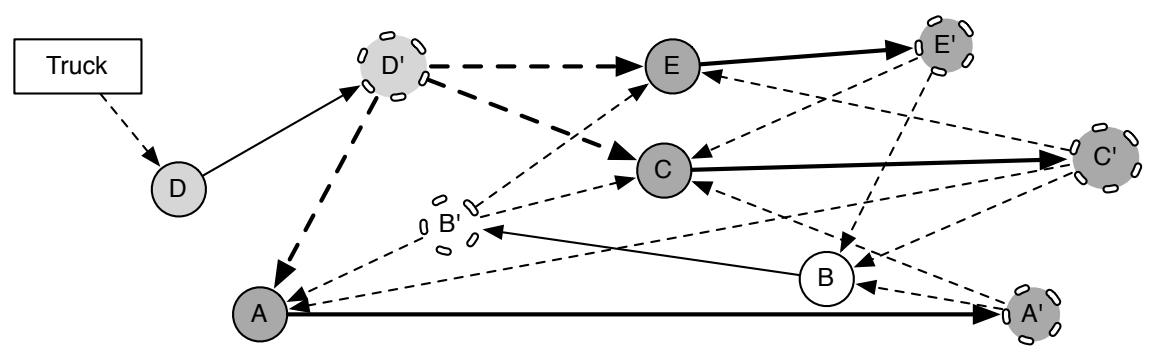

Fig. 2 An abstract representation of behavior of exploration ants on our solution for DPDPTW: a truck has indicated that it will pickup package D and deliver it at D'. Exploration ants are dispatched from D to D' to explore possible future paths from there. At D', information (spread by feasibility ants) about packages $\mathrm{A}, \mathrm{C}$ and $\mathrm{E}$ is known. Thus the exploration ants follow the arrows towards these packages to explore these paths. The explored paths are drawn in bold. Note that in this example the ants consider only one package ahead (one hop).

multiple exploration ants, which provide a number of possible paths to the truck agent. The truck agents chooses its path by using a heuristic with the following objectives, in descending order of importance:

1. Delivering all packages without violating time windows.

2. If it is not possible to comply to a time window, minimization of lateness.

3. Minimization of the total distance traveled by the trucks.

When multiple trucks are planning the delivery of the same package, a conflict arises. This conflict is resolved by using a similar heuristic for comparing paths, packages can decide to be planned in a path of higher quality.

3. Intention Ants. Truck agents delegate the task of negotiation and reservation with the package agent to intention ants. Once a truck decides to go to a package, intention ants are regularly sent to the package agents to make and continually reinstate a reservation.

Agent decision logic. As described earlier, truck agents make decisions about path selection by using heuristics. This decision determines the order at which packages should be serviced. In Figure 3 a heuristic and three helper functions are introduced. The implementation conforms to the heuristic objectives and is used in the experiments described in section 4 The pseudocode first considers time window violations, then lateness followed by distance traveled.

In order to deal with dynamic environments, ants are sent out periodically. Package information and reservations are expired after a certain period of time by examining their time stamps. The adaptation rate controls these aspects of our solution.

Our solution embraces the dynamism in the problem by periodically disseminating local information, expiring outdated information, and by collecting the recent information through ants. We are able to tune adaptation rate which determines 


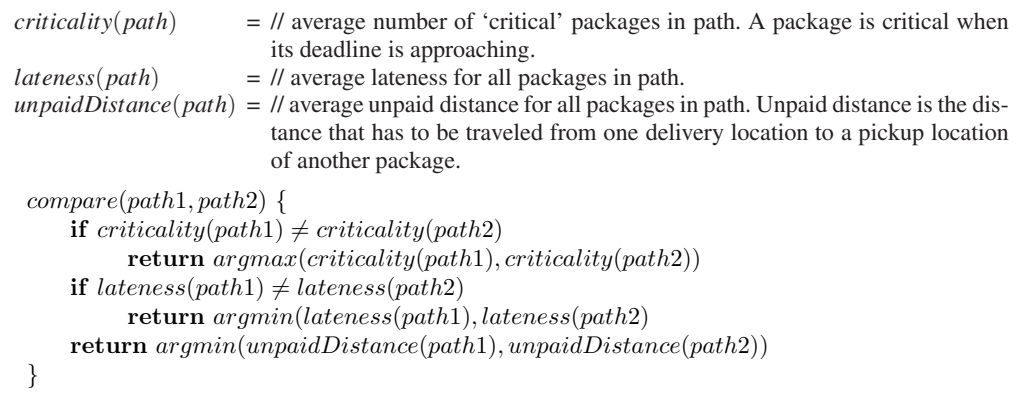

Fig. 3 Agent decision heuristic.

the solution's responsiveness to changes in the environment. Since agents in our solution can make decisions only based on local information, the solution is decentralized. This avoids the requirement of maintaining centralized global information which allows the solution to be scalable.

\section{Experiments}

In this section we present the setup and results of two simulation experiments. The goals of our experiments are to evaluate the adaptability and scalability of our solution in a simulation.

Adaptability experiment. In this experiment, we investigate the performance of our solution in environments with a different degree of dynamism. We compare two instances of our solution on an identical problem scenario: 'fast adaptation' and 'slow adaptation' with a different value for their adaptation rate. As indicated by the name, 'fast adaptation' adapts faster to dynamism in the problem compared to 'slow adaptation' which corresponds to a more static solution.

Scalability experiment. This experiment is designed to investigate whether our solution 'scales', i.e. a scaled problem should result in a proportionally decrease in performance of our solution. Two scenarios of different scale are used, one 'small' scenario of the Leuven city map and one 'big' scenario using a map of Brussels.

Experiment setup. In our experiments the road structure is based on OpenStreetMap1 data. We use the road structure of two cities in Belgium: Brussels and Leuven. The adaptability experiment uses only the Leuven map, while the scalability experiment uses both maps. Each experiment is done for a range of trucks from 7 until 14. This range of trucks is chosen since for less than 7 trucks, the solution cannot handle the packages properly which results in inferior performance. Above this range, the performance of the solution does not improve significantly anymore. Each experiment is

\footnotetext{
1 Www. openstreetmap.org
} 
repeated 20 times with uniform randomly distributed package arrival time and pickup and delivery location. A package deadline is set to a multiple of the travel time to deliver the package ( package deadline $=15 \cdot$ deliverTime). All packages are made available within 12 hours after the start time. A simulation is finished when all packages are delivered. This means that each simulation will take at least 12 hours of simulation time, a maximum duration depends solely on the performance of the solution. The experiments were run using a simulator developed specifically for this project. Computing a simulation generally takes about 30 seconds to 5 minutes, depending on the size of the map and the other experimental settings.

In the following table the Leuven and Brussels scenarios are characterized.

\begin{tabular}{|l||l|l||r|}
\hline & Leuven & Brussels & Factor \\
\hline \hline Number of packages & 200 & 800 & 4 \\
\hline Number of trucks (n=7..14) & $n$ & $4 \cdot \mathrm{n}$ & 4 \\
\hline Accumulated road length (two way) & $968.09 \mathrm{~km}$ & $12946.78 \mathrm{~km}$ & 13.37 \\
\hline $\begin{array}{l}\text { Mean distance between package origin and } \\
\text { destination }\end{array}$ & $8.94 \mathrm{~km}$ & $28.75 \mathrm{~km}$ & 3.22 \\
\hline
\end{tabular}

In order to compare the results between these two maps, we increased the package delivery deadline for the Brussels scenario by 3.22, based on the difference in distance between package origin and destination as shown in the table above.

Experiment results. For both experiments the same two dependent variables are measured:

lateness The average lateness of the delivery of all packages (in minutes). distance The average distance traveled by all trucks (in kilometer).

Figure 4(a) shows lateness of both the fast adapting and the slow adapting solution. On average the fast adapting solution performs better as compared to the slow adapting solution. The variance of the fast adapting solution is smaller, indicating that its performance is more predictable. This is to be expected because the slow adapting (more static) solution generally suffers from any dynamism in the problem, which causes a greater variance in outcome. This variance can be explained by the global behavior of MASs which depends on the interaction of many agents. However, the better performance of the fast adapting solution has a cost of communication overhead since it sends ants more frequently. Part of our future work is to investigate how to make a balance between these two factors. Figure 4(b) shows that the fast adapting solution consistently performs better with respect to distance traveled compared to the slow adapting solution. The outcome of this experiment can also be used for determining the preferred number of trucks in a scenario given an objective.

Figure 5(a) shows the average package lateness for the scalability experiment. It can be seen that for the Brussels scenario the lateness is descending with the increase in number of trucks. It is shown that starting at about 40 trucks the lateness can be reduced close to 0 for the Brussels scenario. This shows that to multiply the number of trucks with 4 is not enough, which can be explained by the differences in topology 


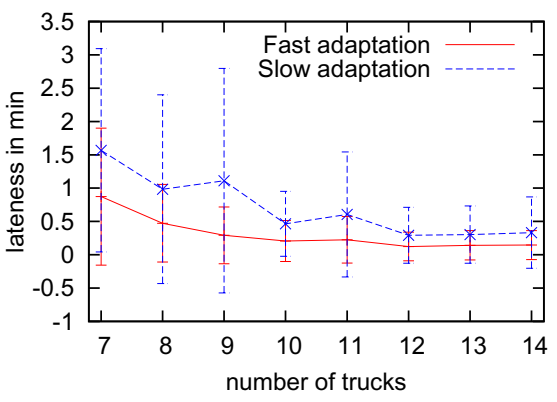

(a) Average package lateness.

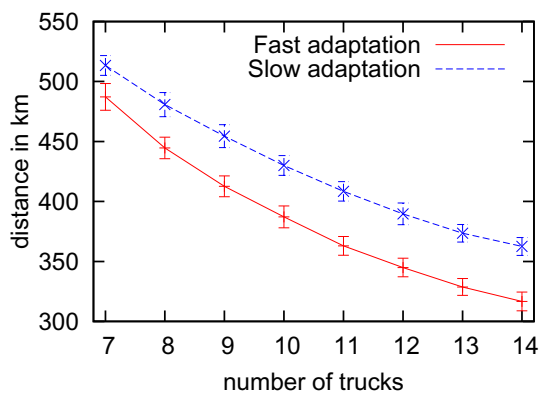

(b) Average distance traveled per truck.

Fig. 4 Adaptability experiment: comparison of results for the solution with fast adaptation and slow adaptation.

between the two maps. Note that the results for the Leuven map are actually the same as the fast adapting solution which was plotted in Figure 4(a) Figure 5(b) shows the average distance traveled per truck for the scalability experiment. The difference in traveled distance between the Leuven and Brussels map is about a factor of 2. In both Figure 5(a) and Figure 5(b) a consistent increase in performance is shown. This illustrates that when the scale of the problem is increased, our solution performs well with respect to lateness and distance traveled.

Analyzing the scalability of a solution is complicated since it is nearly impossible to properly scale a problem instance using a single factor. In our experiments, we scaled the problem by taking a similar but bigger city and choosing an arbitrary scale factor. The experiment results indicate that our solution has consistent performance on both scales.

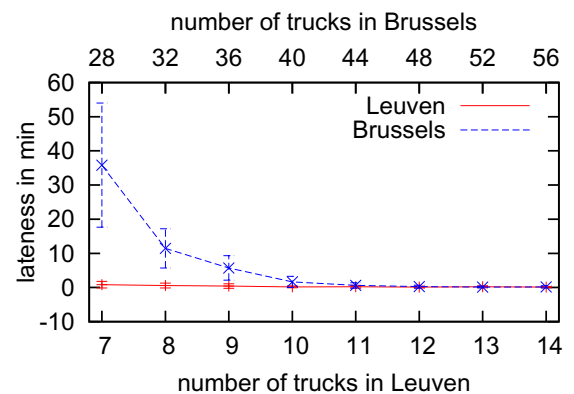

(a) Average package lateness.

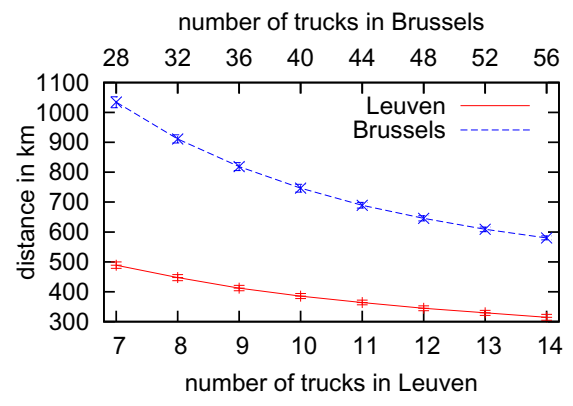

(b) Average distance traveled per truck.

Fig. 5 Scalability experiment: comparison of results for the Leuven and Brussels maps. 


\section{Conclusions}

In this paper, we presented a delegate MAS solution for solving dynamic PDP. The agents interact through lightweight ants that explore a local environment and perform actions on behalf of their originators. There are two main features of the approach. First, the control is decentralized. Agents make decisions based on their localized view. This offers scalability, as it is not required to maintain accurate global information like in previous approaches. Second, agents coordinate with each other through the environment, which enables the solution to dynamically adapt to the changing environment.

We applied our approach to a DPDPTW and performed a set of experiments. Simulation results show that our approach is capable of adapting to a dynamic environment. The solution shows consistent performance in terms of lateness and distance traveled, supporting the claim on scalability. If the necessary infrastructure is available (i.e. smart devices with communication capabilities, at pickup and target locations, and at vehicles), the solution can be physically deployed and operated as a distributed software system.

In our future experiments, we will incorporate additional problem constraints covering additional details i.e. traffic jams or failures encountered by trucks, different patterns of request arrivals, dynamically changing request time windows etc.. We already performed experiments for truck failures, which showed encouraging results. We also plan to extend this solution for other variants of PDPs.

\section{References}

1. Caridi, M., Cavalieri, S.: Multi-agent Systems in Production Planning and Control: an Overview. Production Planning and Control 15(2), 106-118 (2004)

2. Glaschenko, A., Ivaschenko, A., Rzevski, G., Skobelev, P.: Multi-Agent Real Time Scheduling System for Taxi Companies. In: Autonomous Agents and Multi-Agent Systems, AAMAS (2009)

3. Dorer, K., Calisti, M.: An Adaptive Solution to Dynamic Transport Optimization. In: Proc. of 4th Int. Conf. on Autonomous Agents and Multi-Agent Systems, AAMAS (2005)

4. Parragh, S.N., Doerner, K.F., Harti, R.F.: A survey on PDP Part II: Transportation between Pickup and Delivery Locations. Journal fr Betriebswirtschaft 58(2), 81-117 (2008)

5. Eksioglu, B., Vural, A.V., Reisman, A.: The Vehicle Routing Problem: A Taxonomic Review. Computers and Industrial Engineering 57(4), 1472-1483 (2009)

6. Berbeglia, G., Cordeau, J.F., Laporte, G.: Dynamic Pickup and Delivery Problems. European Journal of Operational Research 202, 8-15 (2010)

7. Hentenryck, P.V., Bent, R.W.: Online Stochastic Combinatorial Optimization. Operations Research 52, 977-987 (2004)

8. Rizoli, A.E., Montemanni, R., Lucibello, E., Gambardella, L.M.: Ant Colony Optimization for Real World Vehicle Routing Problems. Swarm Intelligence 1(2), 135-151 (2007)

9. Kouki, Z., Chaar, B.F., Ksouri, M.: Extended CNP Framework for the dynamic Pickup and Delivery Problem Solving. In: Artificial Intelligence and Innovations III 296 (2009) 
10. Fischer, K., Muller, J., Pischel, M.: Cooperative Transportation Scheduling: An Application domain for DAI. Journal of Applied Artificial Intelligence (1995)

11. Kozlak, J., Creput, J.C., Hilaire, V., Koukam, A.: Multi-agent Approach to Dynamic PDP with Uncertain Knowledge about Future Transport Demands. Fundamenta Informaticae 71 (2006)

12. Holvoet, T., Valckenaers, P.: Exploiting the Environment for Coordinating Agent Intentions. In: Weyns, D., Van Dyke Parunak, H., Michel, F. (eds.) E4MAS 2006. LNCS (LNAI), vol. 4389, pp. 51-66. Springer, Heidelberg (2007)

13. Claes, R., Holvoet, T.: Maintaining a Distributed Symbiotic Relationship using Delegate Multi agent Systems. In: Proc. of Winter Simulation Conference (2010)

14. Weyns, D., Holvoet, T., Helleboogh, A.: Anticipatory Vehicle Routing Using Delegate Multi-Agent Systems. In: Proc. of IEEE intelligent Transportation Systems Conference (2007)

15. Gendreau, M., Guertin, F., Potvin, J.Y., Seguin, R.: Neighborhood Search Heuristics for a Dynamic Vehicle Dispatching Problem with Pick-ups and Deliveries. Transportation Research Part C 14, 157-174 (2005)

16. Psaraftis, H.N.: Dynamic Vehicle Routing: Status and Prospects. Annals of Operations Research 61, 143-164 (1995)

17. Gutenschwager, K., Niklaus, C., Vob, S.: Dispatching of an Electric Monorail System: Applying Metaheuristics to an Online PDP. Transportation Science 38, 434-446 (2004)

18. Powell, W.B., Towns, M.T., Marar, A.: On the Value of Optimal Myopic Solutions for Dynamic Routing and Scheduling Problems in the Presence of user Noncompliance. Transportation Science 34(1), 67-85 (2000)

19. Jennings, N.R.: On Agent-based Software Engineering. Artificial Intelligence 117, 277-296 (2000)

20. Burke, E.K., Kendall, G. (eds.): Search Methodologies: Introductory Tutorials in Optimization and Decision Support Techniques. Springer, Heidelberg (2005)

21. Marrow, P.: Scalability in Multi-Agent Systems: The DIET Project. In: Workshop on Infrastructure for Agents. MAS and Scalable Multi-Agent Systems at Autonomous Agents (2001)

22. Wooldridge, M. (ed.): An Introduction to MultiAgent Systems. Wiley, Chichester (2009)

23. Van Dyke Parunak, H., Brueckner, S.A., Weyns, D., Holvoet, T., Verstraete, P., Valckenaers, P.: E Pluribus Unum: Polyagent and Delegate MAS Architectures. In: Antunes, L., Paolucci, M., Norling, E. (eds.) MABS 2007. LNCS (LNAI), vol. 5003, pp. 36-51. Springer, Heidelberg (2008)

24. Holvoet, T., Weyns, D., Valckenaers, P.: Patterns of Delegate MAS. In: Third IEEE International Conference on Self-Adaptive and Self-Organizing Systems (2009)

25. Weyns, D., Holvoet, T.: From Reactive Robotics to Situated Multiagent Systems: A Historical Perspective on the Role of the Environment in Multiagent Systems. In: Workshop on Engineering Societies in the Agents World, pp. 31-56 (2005) 